\title{
A Primal-Dual Large-update Interior-point Algorithm for Semi-definite Optimization Based on a New Parametric Kernel Function
}

\author{
Dequan Zhao and Mingwang Zhang * \\ College of Science, China Three Gorges University, Yichang, 443002, P. R. China, \\ E-mail: zhaodequandandan@126.com_zmwang@ctgu.edu.cn
}

Received 12 May 2013; Accepted 15 October 2013

Editor: Zhenghai Huang

\begin{abstract}
Based on a new parametric kernel function, this paper presents a primaldual large-update interior-point algorithm (IPM) for semi-definite optimization (SDO) problems. The new parametric function is neither self-regular function nor the usual logarithmic barrier function. It is strongly convex and possesses some novel analytic properties. We analyse this new parametric kernel function and show that the proposed algorithm has favorable complexity bound in terms of the analytic properties of the kernel function. Moreover, the complexity bound for our large-update IPM is shown to be $O\left(\sqrt{n}(\log n)^{2} \log \frac{n}{\epsilon}\right)$. Some numerical results are reported to illustrate the feasibility of the proposed algorithm.
\end{abstract}

Keywords Semi-definite optimization, Kernel function, Interior-point algorithm, Primal-dual algorithm, Polynomial complexity

DOI: $10.19139 /$ soic.v1i1.8

\section{Introduction}

Throughout this paper we deal with complexity analysis of large-update primaldual IPMs for solving SDO problems. SDO problems are one of the fastest developing branches of mathematical programming. They are convex optimization problems over the intersection of an affine set and the cone of positive semidefinite matrices. Since the Karmarkar's path-breaking paper [6], a great many of

*Correspondence to: Mingwang Zhang. Email: zmwang@ctgu.edu.cn.

ISSN 2310-5070 Copyright (c) 2013 International Academic Press 
researchers such as Nesterov, Nemirovsky [7], Todd [12] and so on have proposed and analyzed various IPMs for convex optimization and SDO. Many plentiful results have been reported $[11,14]$. One main reason for this increasing interest is that SDO problems have found numerous applications in various fields, such as continuous and combinational optimization, structural design, statistics and electrical engineering. Furthermore, lately efficient new algorithms, IPMs have shown their powers in solving linear optimization (LO) and large classes of other optimization problems.

In this paper, we deal with so-called primal-dual IPMs which are most efficient from a computational point of view. We consider the standard form of SDO problems:

$$
\begin{gathered}
(P) \quad \min C \bullet X, \\
\text { subject to } A_{i} \bullet X=b_{i}, \quad i=1,2, \cdots, m, \\
X \succeq 0 .
\end{gathered}
$$

and its dual problem

$$
\begin{gathered}
(D) \quad \max b^{T} y, \\
\text { subject to } \sum_{i=1}^{m} y_{i} A_{i}+S=C, \\
S \succeq 0 .
\end{gathered}
$$

where each $A_{i} \in S^{n}$ and they are further assumed to be linearly independent, $b \in R^{m}, C \in S^{n}$. Nesterov and Nemirovsky devote themselves to the study of self-concordant barrier function and in 1988 they came to a conclusion that IPMs can, in principle, apply to all the convex optimization problems. Since SDO is an extension of LO, several IPMs designed for LO have been successfully extended to SDO. Among all the methods, primal-dual IPMs are of highly efficient both in theory and in practice. A significant work in this direction is the paper of Nesterorv and Todd [8], they showed that the primal-dual IPM for LO maintains its theoretical efficiency when the nonnegativity constrains in LO are placed by a convex cone, as long as the cone is self-scaled. The most of polynomial time primal-dual IPMs for LO use the classical logarithmic barrier function

$$
\psi_{c}(t)=\frac{1}{2}\left(t^{2}-1\right)-\log t
$$

J.Peng et al. [9, 10] introduced so-called self-regular kernel functions and proposed primal-dual IPM for LO based on self-regular function, and also extended the approach to SDO. The complexity bounds obtained by them are currently the best known bounds, namely, $O\left(\sqrt{n} \log n \log \frac{n}{\epsilon}\right)$, and $O\left(\sqrt{n} \log \frac{n}{\epsilon}\right)$, for large-update IPMs and small-update IPMs for LO and SDO respectively. However, to our best knowledge there is still a gap between the practical behavior 
of these algorithms and the theoretical performance results. In practice, largeupdate IPMs are much more efficient than the so-called small-update IPMs. And the vital gap between practice and theory has been referred to as the irony of IPMs [9, 1]. Subsequently, Bai et al. [1] developed a class of primal-dual IPMs for LO based on elibigle barrier function and obtained the same favorable iteration bounds for the algorithms [9]. Later, other kernel functions were also put forward $[1,3]$. Recently, [16] developed some new analysis tools for convex quadratic semi-definite optimization based on a new kernel function with iteration bound $O\left(\sqrt{n}(\log n)^{2} \log \frac{n}{\epsilon}\right)$. The bound is better than that by the classical primal-dual IPMs based on logarithmic barrier function and some other kernel functions.

Motivated by [16], in this paper we propose a primal-dual large-update IPM for SDO based on the kernel function:

$$
\psi(t)=\frac{t^{2}-1}{2}-(t-1) e^{p\left(\frac{1}{t}-1\right)}, \quad t>0 .
$$

where $1 \leq p<2$ is a parameter. This function $\psi(t)$ is a parameterized version of kernel function [16], however the analysis tools in [16] is no longer applicable. We do some analysis about our new parametric kernel function $\psi(t)$ and design the corresponding primal-dual IPM based on $\psi(t)$. And the primal-dual IPM has favorable complexity bound in terms of the analytic properties of $\psi(t)$. The best complexity for SDO based on kernel function has been known as $O\left(\sqrt{n}(\log n) \log \frac{n}{\varepsilon}\right)$, but it is worth mentioning that the kernel function $\psi(t)$ cannot be presented in other papers. Numerical results also indicated its favorable practical behavior.

The paper is organized as follows. In Section 2, we first describe matrix functions used in later sections. And then we briefly recall the basic concepts of IPMs for SDO. A generic primal-dual IPM for SDO is described in Section 3. In Section 4, we present the properties of $\psi(t)$ and study the matrix function $\psi(V)$ and $\Psi(V)$. We analyze the algorithm and derive the complexity bound for SDO in Section 5. Some numerical results are provided in Section 6. Finally, some concluding remarks follow in Section 7.

Some notions used throughout the paper are as follows. $R^{n}, R_{+}^{n}$ and $R_{++}^{n}$ denote the set of vectors with $n$ components, the set of $n$-dimensional nonnegative vectors and the set of $n$-dimensional positive vectors, respectively. $\|\cdot\|$ denotes the Frobenius norm for matrices and 2-norm for vectors. $S^{n}, S_{+}^{n}$ and $S_{++}^{n}$ denote the cone of symmetric, symmetric positive semi-definite and symmetric positive define $n \times n$ matrices, respectively. The classical Löwner partial order " $\succeq$ " on positive semi-definite (or positive definite) matrices means $A \succeq B$ (or $A \succ B$ ) if $A-B$ is positive semi-definite (or positive definite). The matrix inner product $A \bullet B=\operatorname{Tr}\left(A^{T} B\right)$. For any symmetric positive definite matrix $Q \in S_{++}^{n}$, the expression $Q^{\frac{1}{2}}$ (or $\sqrt{Q}$ ) denotes its symmetric square root. When $\lambda$ is a vector we denote the diagonal matrix $\Lambda$ with entries $\lambda_{i}$ by $\operatorname{diag}(\lambda)$. For any $V \in S_{++}^{n}$, we 
denote by $\lambda(V)$ the vector of eigenvalues of $V$ arranged in non-increasing order, namely, $\lambda_{1}(V) \geq \lambda_{2}(V) \geq \cdots \geq \lambda_{n}(V)$. Finally, if $g(x) \geq 0$ is a real valued function of a real nonnegative variable, we write $g(x)=O(x)$ if $g(x) \leq c x$ for some positive constant $c$ and $g(x)=\Theta(x)$ if $c_{1} x \leq g(x) \leq c_{2} x$ for two positive constants $c_{1}$ and $c_{2}$.

\section{Preliminaries}

In this section, we recall some basic definitions and several related conclusions about matrix functions which will be used for analyzing the algorithm.

\section{Theorem 2.1}

(Spectral theorem for symmetric matrices in [5]) The real $n \times n$ matrix $A$ is symmetric if and only if there exists a matrix $U \in R^{n \times n}$ such that $U^{T} U=E$ and $U^{T} A U=\Lambda$ where $\Lambda$ is a diagonal matrix.

The columns $u_{i}$ of $U$ are eigenvectors of $A$, satisfying $A u_{i}=\lambda_{i} u_{i}, \quad i=$ $1,2, \cdots, n$, where $\Lambda$ is a diagonal entry of $\Lambda$. Now we prepare to show how a matrix function can be obtained from $\psi(t)$.

\section{Definition 2.2}

(Definition 2.2 in [13]) Let $V \in S_{++}^{n}$ be any symmetric $n \times n$ matrix and

$$
V=Q^{T} \operatorname{diag}(\lambda(V)) Q=Q^{T} \operatorname{diag}\left(\lambda_{1}(V), \lambda_{2}(V), \cdots, \lambda_{n}(V)\right) Q,
$$

where Q is any orthonormal matrix that diagonalizes V. Let $\psi(t)$ be defined as in (2). The matrix function $\psi(V): S_{++}^{n} \rightarrow S^{n}$ is defined by

$$
\psi(V)=Q^{T} \operatorname{diag}\left(\psi\left(\lambda_{1}(V)\right), \psi\left(\lambda_{2}(V)\right), \cdots, \psi\left(\lambda_{n}(V)\right)\right) Q .
$$

The matrix function $\psi(V)$ depends only on the restriction of $\psi(t)$ to the spectrum of $V$ (the set of eigenvalues). Suppose that $\psi(t)$ is twice differentiable for $t>0$. The derivatives $\psi^{\prime}(t)$ and $\psi^{\prime \prime}(t)$ are well defined and obtained by replacing $\psi\left(\lambda_{i}(V)\right)$ in (4) by $\psi^{\prime}\left(\lambda_{i}(V)\right)$ and $\psi^{\prime \prime}\left(\lambda_{i}(V)\right)$, respectively, for each $1 \leq i \leq n$.

\section{Definition 2.3}

(Definition 2.3 in [13]) The matrix function $\Psi(V): S_{++}^{n} \rightarrow R_{+}$is defined by

$$
\Psi(V):=\operatorname{Tr}(\psi(V))=\sum_{i=1}^{n} \psi\left(\lambda_{i}(V)\right) .
$$

Where $\psi(V)$ is given by (4). The matrix function $\Psi(V)$ will play an important role in the following analysis, moreover we call both $\psi(V)$ and $\Psi(V)$ matrix barrier function, which is determined by kernel function $\psi(t)$. 
In the sequel, we state two inequalities, which are used in the following analysis. If $M, N \in S^{n}$, then

$$
|\operatorname{Tr}(M N)| \leq\left|\lambda_{1}(M)\right| \sum_{i=1}^{n}\left|\lambda_{i}(N)\right| .
$$

Furthermore, if $M_{1} \preceq M_{2}$ and $N \succeq 0$, then

$$
\operatorname{Tr}\left(M_{1} N\right) \preceq \operatorname{Tr}\left(M_{2} N\right) .
$$

\section{Definition 2.4}

(Definition 2.5 in [13]) A matrix $M(t)$ is said to be matrix of function if each entry of $M(t)$ is a function of t, i.e., $M(t)=\left[M_{i j}(t)\right]$.

The usual concept of continuity, integrability and differentiability can be naturally extended to matrices of functions by interpreting them entry-wise. Suppose that $M(t)$ and $N(t)$ be two matrices of function which are differentiable. Then we have

$$
\begin{aligned}
& \frac{d}{d t} M(t)=\left[\frac{d}{d t} M_{i j}(t)\right]=M^{\prime}(t), \\
& \begin{aligned}
\frac{d}{d t} \operatorname{Tr}(M(t))= & \operatorname{Tr}\left(M^{\prime}(t)\right), \\
\frac{d}{d t} \operatorname{Tr}(\psi(M(t))) & =\operatorname{Tr}\left(\psi^{\prime}(M(t)) M^{\prime}(t)\right), \\
\frac{d}{d t}(M(t) N(t)) & =\left[\frac{d}{d t} M(t)\right] N(t)+M(t)\left[\frac{d}{d t} N(t)\right] \\
& =M^{\prime}(t) N(t)+M(t) N^{\prime}(t) .
\end{aligned}
\end{aligned}
$$

Remark 2.1

In the rest of the section, when we use the function $\psi(\cdot)$ and its derivatives $\psi^{\prime}(\cdot)$ and $\psi^{\prime \prime}(\cdot)$, these denote matrix function if the argument is matrix and a univariate function if the argument is in $R_{+}$.

Without loss of generality, we assume that both $(P)$ and $(D)$ satisfy the interiorpoint condition (IPC), i.e., there exists a strictly feasible pair $\left(X^{0} \succ 0, y^{0}, S^{0} \succ\right.$ $0)$ such that

$$
\begin{gathered}
A_{i} \bullet X^{0}=b_{i}, \quad X^{0} \succ 0, \quad i=1,2, \cdots, m, \\
\sum_{i=1}^{m} y_{i} A_{i}+S^{0}=C, \quad S^{0} \succ 0 .
\end{gathered}
$$

If $\left(X^{0} \succeq 0, \quad y^{0}, \quad S^{0} \succeq 0\right)$ is an optimal solution of $(P)$ and $(D)$, we have $X^{0} S^{0}=0$, which is the so-called complementary condition. And this can be 
viewed as the extension of linear programming complementary condition. Then the optimality conditions for the pair of problem $(P)$ and $(D)$ are

$$
\begin{aligned}
& A_{i} \bullet X=b_{i}, \quad i=1,2, \cdots, m, \quad X \succeq 0, \\
& \sum_{i=1}^{m} y_{i} A_{i}+S=C, \quad S \succeq 0 . \\
& X S=0 .
\end{aligned}
$$

The basic idea of primal-dual IPMs is to replace the third equation in the system (12) by the parameterized equation $X S=\mu E$, with $\mu>0$, and $E$ is the $n \times n$ unit matrix. The system of relaxed optimality conditions is presented as follows.

$$
\begin{aligned}
& A_{i} \bullet X=b_{i}, \quad i=1,2, \cdots, m, \quad X \succeq 0, \\
& \sum_{i=1}^{m} y_{i} A_{i}+S=C, \quad S \succeq 0, \\
& X S=\mu E .
\end{aligned}
$$

Since the IPC holds and the matrices $A_{i}$ are linearly independent, the system (13) has a unique solution. It is denoted as $(X(\mu), y(\mu), S(\mu))$ and we call $X(\mu)$ the $\mu$-center of $(P)$ and $(y(\mu), S(\mu))$ the $\mu$-center of $(D)$, respectively. The set $\{(X(\mu), y(\mu), S(\mu)): \mu>0\}$ gives a homotopy path, which is called the central path of $(P)$ and $(D)$. If $\mu \rightarrow 0$ then the limit of the central path exists. And the limit point satisfies the complementarity condition, hence it naturally yields optimal solutions for both $(P)$ and $(D)$.

The core idea of primal-dual IPMs is to follow the central path and to approach the optimal set of SDO problems by $\mu \rightarrow 0$. Newton method applied to system (13) produces the following system:

$$
\left\{\begin{array}{l}
A_{i} \bullet \Delta X=0, \quad i=1,2, \cdots, m, \\
\sum_{i=1}^{m} \Delta y_{i} A_{i}+\Delta S=0 \\
\Delta X+X \Delta S S^{-1}=\mu S^{-1}-X .
\end{array}\right.
$$

A decisive observation for SDO is that $\Delta X$ in the above Newton system is not necessary symmetric, although $\Delta S$ is symmetric. Many researchers have proposed several methods for symmetrizing the third equation in (14) such that the resulting new system has a unique symmetric solution. In this paper we use the NT symmetrization scheme, from which the NT direction is derived. The main idea of NT symmetrization scheme transfers the primal variable $X$ and the dual variable $S$ into the same space: V-space. The term $X \Delta S S^{-1}$ in the third equation 
is replaced by $P \Delta S P^{T}$. Let

$$
P=X^{\frac{1}{2}}\left(X^{\frac{1}{2}} S X^{\frac{1}{2}}\right)^{-\frac{1}{2}} X^{\frac{1}{2}}=S^{-\frac{1}{2}}\left(S^{\frac{1}{2}} X S^{\frac{1}{2}}\right)^{\frac{1}{2}} S^{-\frac{1}{2}}, D=P^{\frac{1}{2}} .
$$

The matrix $D$ can be used to scale $X$ and $S$ to the same matrix $V$ :

$$
V:=\frac{1}{\sqrt{\mu}} D^{-1} X D^{-1}=\frac{1}{\sqrt{\mu}} D S D .
$$

Obviously, the matrices $D$ and $V$ are symmetric and positive define. We have

$$
V^{2}=\frac{1}{\mu} D^{-1} X S D
$$

Let us further define

$$
\bar{A}_{i}:=\frac{1}{\sqrt{\mu}} D A_{i} D, i=1, \cdots, m, D_{X}:=\frac{1}{\sqrt{\mu}} D^{-1} \Delta X D^{-1}, D_{S}:=\frac{1}{\sqrt{\mu}} D \Delta S D .
$$

Then the (scaled) NT search direction $\left(D_{X}, \Delta y, D_{S}\right)$ satisfies the following system

$$
\left\{\begin{array}{l}
A_{i} \bullet D_{X}=0, \quad i=1,2, \cdots, m, \\
\sum_{i=1}^{m} \Delta y_{i} \bar{A}_{i}+D_{S}=0 \\
D_{X}+D_{S}=V^{-1}-V .
\end{array}\right.
$$

It is not difficult to verify that the right-hand side in the third equation in (19) equals minus the derivative of the classical logarithmic barrier function (1). Thus replace the $V^{-1}-V$ for $-\psi^{\prime}(V)$ and let $-\psi^{\prime}(V):=D_{V}$, we have the following systems

$$
\left\{\begin{array}{l}
A_{i} \bullet D_{X}=0, \quad i=1,2, \cdots, m, \\
\sum_{i=1}^{m} \Delta y_{i} \bar{A}_{i}+D_{S}=0 \\
D_{X}+D_{S}=-\psi^{\prime}(V)=D_{V} .
\end{array}\right.
$$

From the above system we can easily have

$$
\sum_{i=1}^{m} \Delta y_{i} \bar{A}_{i} \cdot \bar{A}_{j}=-\bar{A}_{j} \cdot D_{S}=-\bar{A}_{j} \cdot D_{V}, \quad j=1,2, \cdots, m,
$$

According to the assume that $A_{i}$ is independent, we can conclude that (21) has a unique solution $\Delta y$ and then plugging $\Delta y$ into (20), we immediately get $D_{S}$ and $D_{X}$. At last we can get the direction $(\Delta X, \Delta y, \Delta S)$ from (18). From the analysis 
we argue that the matrix function $\Psi(V)$ determines an IPMs in a natural way. It is obviously that $D_{X}$ and $D_{S}$ are orthogonal, i.e,

$$
\operatorname{Tr}\left(D_{X} D_{S}\right)=\operatorname{Tr}\left(D_{S} D_{X}\right)=0 .
$$

Thus we obtain that

$$
\left(\left\|D_{X}+D_{S}\right\|\right)^{2}=\left\|D_{X}\right\|^{2}+\left\|D_{S}\right\|^{2}=\left\|\psi^{\prime}(V)\right\|^{2} .
$$

In the analysis of the algorithm we also use the norm-based proximity measure $\delta(V)$, which is defined by

$$
\delta(V):=\frac{1}{2}\left\|\psi^{\prime}(V)\right\|=\frac{1}{2} \sqrt{\sum_{i=1}^{n}\left(\psi^{\prime}\left(\lambda_{i}(V)\right)^{2}\right.} .
$$

And we have

$$
D_{X}=D_{S}=0_{n \times n} \Leftrightarrow \delta(V)=0_{n \times n} \Leftrightarrow V=E \Leftrightarrow \Psi(V)=0 .
$$

If and only if $X S=\mu E$. That is $\Psi(V)=0$ if and only if $(X, y, S)=$ $(X(\mu), y(\mu), S(\mu))$, otherwise $\Psi(V)>0$. It is clear from the description that the closeness of $(X, y, S)$ to $(X(\mu), y(\mu), S(\mu))$ is measured by the valued of $\Psi(V)$. If $(X, y, S) \neq(X(\mu), y(\mu), S(\mu))$ then $(\Delta X, \Delta y, \Delta S)$ is nonzero. By taking a step along the search direction, with the step size $\alpha$ defined by some line search rules, we construct a new triple $(X, y, S)$ according to

$$
X_{+}=X+\alpha \Delta X, y_{+}=y+\alpha \Delta y, S_{+}=S+\alpha \Delta S .
$$

\section{A generic primal-dual algorithm for SDO}

We can now describe the algorithm briefly. According to the definition of the matrix $V$, it is determined by the current iterates $(X, S)$ and the center parameter $\mu$. Thus $\Psi(X, S ; \mu)$ in the algorithm is another expression of the matrix function, we denote $\Psi(V):=\Psi(X, S ; \mu)$. Given a kernel $\psi(t)$, we can get a matrix barrier function $\Psi(V)$. It is clear from the afore-mentioned description that the closeness of $(X, y, S)$ is measured by the value of $\Psi(V)$. As for a threshold parameter given in advance, if $\Psi(V) \leq \tau,(X, y, S)$ is "close" enough to the current center path, we start a new outer iteration by updating center parameter $\mu:=(1-\theta) \mu$, otherwise we enter an inner iteration. The choice of the step size $\alpha$ and the barrier update parameter $\theta$ play an important role both in theory and practice of IPMs. The generic form of our algorithm is shown as follows: 


\section{IPMs for SDO Based on a New Parametric Kernel Function}

\section{Input:}

A threshold parameter $\tau \geq 1$;

an accuracy parameter $\epsilon>0$;

a fixed barrier update parameter $\theta, 0<\theta<1$;

a strictly feasible pair $\left(X^{0}, S^{0}\right)$ and $\mu^{0}=1$ such that $\Psi\left(X^{0}, S^{0} ; \mu^{0}\right) \leq \tau$.

begin

$$
\begin{aligned}
& X:=X^{0} ; y:=y^{0} ; S:=S^{0} ; \mu:=\mu^{0} ; \\
& \text { while } n \mu \geq \epsilon \text { do } \\
& \text { begin } \\
& \mu:=(1-\theta) \mu ; \\
& \quad \text { while } \Psi(X, S ; \mu)>\tau \text { do } \\
& \quad \text { begin }
\end{aligned}
$$

Solve system (20) and use (18) to obtain $(\Delta X, \Delta y, \Delta S)$;

Determine a suitable step size $\alpha$;

Update $(X, y, S):=(X, y, S)+\alpha(\Delta X, \Delta y, \Delta S)$.

$$
\begin{aligned}
& V:=\frac{1}{\sqrt{\mu}}\left(D^{-1} X S D\right)^{\frac{1}{2}} \text {. } \\
& \text { end }
\end{aligned}
$$

end

end

\section{Properties of the Kernel (Barrier) Function}

In this section, we study some basic properties of our kernel function $\psi(t)$ and the corresponding barrier function $\Psi(V)$. As we need the first three derivatives of $\psi(t)$ with respect to $t$ frequently, we provide them as follows:

$$
\begin{aligned}
& \psi^{\prime}(t)=t-\left(1-\frac{p(t-1)}{t^{2}}\right) e^{p\left(\frac{1}{t}-1\right)} \\
& \psi^{\prime \prime}(t)=1+\frac{\left(2 p-p^{2}\right) t+p^{2}}{t^{4}} e^{p\left(\frac{1}{t}-1\right)}, \\
& \psi^{\prime \prime \prime}(t)=-\frac{3 p(2-p) t^{2}+p^{2}(6-p) t+p^{3}}{t^{6}} e^{p\left(\frac{1}{t}-1\right)}, 1 \leq p<2 .
\end{aligned}
$$

It follows that $\psi(1)=\psi^{\prime}(1)=0$, and it is also quite straightforward to verify that

$$
\lim _{t \rightarrow 0^{+}} \psi(t)=\lim _{t \rightarrow+\infty} \psi(t)=+\infty
$$


Thus the univariate function $\psi(t)$ is a kernel function. In order to analyze the algorithm and compute the complexity bound of our algorithm, some properties of the new kernel function are given in the following lemma.

\section{Lemma 4.1}

Let the function $\psi(t)$ be defined as in (2). Then we have:

(a) $\psi^{\prime \prime}(t)>1, \quad t>0$,

(b) $t \psi^{\prime \prime}(t)+\psi^{\prime}(t)>0, \quad t<1$,

(c) $\psi^{\prime \prime \prime}(t)<0, \quad t>0$,

\section{Proof}

We first prove that $(a)$ holds. From (27), we have

$$
\psi^{\prime \prime}(t)=1+\frac{\left(2 p-p^{2}\right) t+p^{2}}{t^{4}} e^{p\left(\frac{1}{t}-1\right)}
$$

Considering

$$
f_{1}(t):=\left(2 p-p^{2}\right) t+p^{2}=p(2-p) t+p^{2},
$$

it is obvious that $f_{1}(t)$ is monotonically increasing and $f_{1}(0)=p^{2}>0$, hence $\psi^{\prime \prime}(t)>1$ holds for all $t>0$. Furthermore, for proving $(b)$. We first have:

$$
t \psi^{\prime \prime}(t)+\psi^{\prime}(t)=2 t+\frac{-t^{3}+p t^{2}+\left(p-p^{2}\right) t+p^{2}}{t^{3}} e^{p\left(\frac{1}{t}-1\right)} .
$$

Since $1 \leq p<2$, we have

$$
-t^{3}+p t^{2}+\left(p-p^{2}\right) t+p^{2} \geq-t^{3}+p t^{2}+p \geq(p-1) t^{3}+p>0,
$$

this prove that $(b)$ is also satisfied. For proving $(c)$, considering the numerator of (28), letting

$$
f_{2}(t):=3 p(2-p) t^{2}+p^{2}(6-p) t+p^{3}
$$

we have

$$
\begin{gathered}
\Delta:=p^{4}(6-p)^{2}-4 p^{4}(6-3 p)=p^{4}\left(p^{2}+12\right)>0, \\
f_{2}(0)=p^{3}>0, \quad 3 p(2-p)>0
\end{gathered}
$$

furthermore the axis of symmetry of $f_{2}(t)$ is

$$
t=-\frac{p^{2}(6-p)}{6 p(2-p)}<0 .
$$

We have $f_{2}(t)>0$, for $t>0$. Thus we obtain the conclusion of $(c)$, i.e. $\psi^{\prime \prime \prime}(t)<$ $0, t>0$. The lemma is proved. 
It has shown from the above lemma that $\psi(t)$ is indeed a strongly convex kernel function and suffice eligibility. We can also get some useful results and properties of $\psi(t)$ from [3]. Due to conditions $\psi(1)=\psi^{\prime}(1)=0$, we can completely describe $\psi(t)$ by its second derivative as follows:

$$
\psi(t)=\int_{1}^{t} \int_{1}^{\zeta} \psi^{\prime \prime}(\xi) d \xi d \zeta .
$$

We have the following lemma about our given kernel function.

\section{Lemma 4.2}

Let $t_{1}>0, t_{2}>0$, we have

$$
\psi\left(\sqrt{t_{1} t_{2}}\right) \leq \frac{1}{2}\left(\psi\left(t_{1}\right)+\psi\left(t_{2}\right)\right)
$$

Proof

This result can be derived by using Lemma 1 in [9], which states that the above inequality holds if and only if $t \psi^{\prime \prime}(t)+\psi^{\prime}(t) \geq 0$ for all $t>0$. Since $\psi^{\prime \prime}(t)>$ $1, \psi(1)=0$, we have $\psi^{\prime}(t)$ is monotonically increasing in $t$. For $t \geq 1$, it follows that $\psi^{\prime}(t)>0$. So we obtain that $t \psi^{\prime \prime}(t)+\psi^{\prime}(t) \geq 0$ for all $t>1$. Furthermore we have already proved that $t \psi^{\prime \prime}(t)+\psi^{\prime}(t) \geq 0$ for all $0<t<1$ in lemma 4.1. Thus we proved the lemma.

We call the property described in Lemma 4.2 exponential convexity.

\section{Lemma 4.3}

(Lemma 3.1 in [1]) When $t_{1} \leq 1 \leq t_{2}$, and $\psi\left(t_{1}\right)=\psi\left(t_{2}\right)$, then

(a) If $\psi^{\prime}\left(t_{1}\right) \leq 0, \psi^{\prime}\left(t_{2}\right) \geq 0$ then $-\psi^{\prime}\left(t_{1}\right) \geq \psi^{\prime}\left(t_{2}\right)$.

(b)For every $\beta \geq 1$, we have

$$
\psi\left(\beta t_{1}\right) \leq \psi\left(\beta t_{2}\right) .
$$

and the inequality hold if and only if $\beta=1$ or $t_{1}=t_{2}$.

We start with the above Lemma to find an upper bound for $\Psi(\beta V)$ in terms of $\Psi(V)$.

\section{Lemma 4.4}

(Proposition 3 in [9]) If $V_{1}, V_{2}$ are the positive definite symmetric matrices then

$$
\Psi\left(\left(V_{1}^{\frac{1}{2}} V_{2} V_{1}^{\frac{1}{2}}\right)^{\frac{1}{2}}\right) \leq \frac{1}{2}\left(\Psi\left(V_{1}\right)+\Psi\left(V_{2}\right)\right) .
$$

\section{Lemma 4.5}

Let the kernel function $\psi(t)$ be defined as in (2). Then we have:

(a) $\frac{1}{2}(t-1)^{2} \leq \psi(t) \leq \frac{1}{2} \psi^{\prime}(t)^{2}$, for all $t>0$,

(b) $\Psi(V) \leq 2 \delta(V)^{2}$, for any $V \succ 0$,

(c) $\|\lambda(V)\| \leq \sqrt{n}+\sqrt{2 \Psi(V)}$, for any $V \succ 0$. 
Proof

We can find a proof of $(a)$ in [2]. To prove $(b)$ of the lemma, using the second inequality of $(a)$, we have

$$
\begin{aligned}
\Psi(V) & =\sum_{i=1}^{n} \psi\left(\lambda_{i}(V)\right) \leq \frac{1}{2} \sum_{i=1}^{n}\left(\psi^{\prime}\left(\lambda_{i}(V)\right)\right)^{2} \\
& =\frac{1}{2}\left\|\psi^{\prime}\left(\lambda_{i}(V)\right)\right\|=2 \delta(V)^{2}
\end{aligned}
$$

Then by using the first inequality of $(a)$, one can obtain

$$
\begin{aligned}
2 \Psi(V) & =2 \sum_{i=1}^{n} \psi\left(\lambda_{i}(V)\right) \geq \sum_{i=1}^{n}\left(\lambda_{i}(V)-1\right)^{2} \\
& =\| \lambda(V)) \|^{2}-2 \sum_{i=1}^{n} \lambda_{i}(V)+n \geq(\|\lambda(V)\|-\|\sqrt{n}\|)^{2} .
\end{aligned}
$$

This completes the proof.

Corollary 4.6

(Corollary 3.4 in [16]) If $V \succ 0$, then

$$
\|V\| \leq \sqrt{n}+\sqrt{2 \Psi(V)} \leq \sqrt{n}+2 \delta(V)
$$

Note that at the start of each outer iteration of the algorithm, just before the update of $\mu$-update, the value of $\Psi(V)$ is increased. And then during the subsequent inner iterations $\Psi(V)$ decreases. So next we derive an estimate for the effect of a $\mu$-update on the value of $\Psi(V)$. We describe the following two important lemmas and we will omit their proofs by just providing appropriate reference.

\section{Lemma 4.7}

(Lemma 3.5 in [16]) If $\beta \geq 1$, then

$$
\psi(\beta t) \leq \psi(t)+\frac{1}{2}\left(\beta^{2}-1\right) t^{2} .
$$

\section{Lemma 4.8}

(Lemma 3.6 in [16]) Let $0 \leq \theta<1$ and $V \succ 0$. Then

$$
\Psi\left(\frac{V}{\sqrt{1-\theta}}\right) \leq \Psi(V)+\frac{\theta}{2(1-\theta)}(2 \Psi(V)+2 \sqrt{2 \Psi(V)}+n) .
$$

By the assumption $\Psi(V) \leq \tau$ just before the update of $\mu$,

$$
\Psi\left(\frac{V}{\sqrt{1-\theta}}\right) \leq \tau+\frac{\theta}{2(1-\theta)}(2 \tau+2 \sqrt{2 n \tau}+n) .
$$


We define

$$
L(n, \theta, \tau)=\tau+\frac{\theta}{2(1-\theta)}(2 \tau+2 \sqrt{2 n \tau}+n) .
$$

Since $\tau=O(n)$ and $\theta=\Theta(1)$, we have

$$
L \triangleq L(n, \theta, \tau)=O(n) .
$$

\section{Analysis of the Algorithm for SDO}

The decrease of the proximity function after one step will be estimated in this section. Let us denote by $V_{+}$the scaled matrix defined by (15) and (16) where the matrix $X$ and $S$ are replaced by $X_{+}=X+\alpha \Delta X, S_{+}=S+\alpha \Delta S$, respectively. Throughout the paper we assume that the step size $\alpha$ is satisfied, where all eigenvalues of the matrices $V+\alpha D_{X}$ and $V+\alpha D_{S}$ are not smaller than zero. It is trivial to verify that $V_{+}^{2}$ is unitarily similar to the matrix $X_{+}^{\frac{1}{2}} S_{+} X_{+}^{\frac{1}{2}}$ and thus to $\left(V+\alpha D_{X}\right)^{\frac{1}{2}}\left(V+\alpha D_{S}\right)\left(V+\alpha D_{X}\right)^{\frac{1}{2}}$. This further implies that the eigenvalue of $V_{+}$are precisely the same as those of the matrix

$$
\bar{V}_{+}:=\left(\left(V+\alpha D_{X}\right)^{\frac{1}{2}}\left(V+\alpha D_{S}\right)\left(V+\alpha D_{X}\right)^{\frac{1}{2}}\right)^{\frac{1}{2}} .
$$

Since the proximity function after one step is defined by $\Psi\left(V_{+}\right)$, from (5) it follows immediately that

$$
\Psi\left(V_{+}\right)=\Psi\left(\bar{V}_{+}\right) .
$$

As in the LO case in [9], we define the gap between the proximity before and after one step as a function of the step size $\alpha$, i.e.,

$$
f(\alpha):=\Psi\left(V_{+}\right)-\Psi(V)=\Psi\left(\bar{V}_{+}\right)-\Psi(V) .
$$

By Lemma 4.4, we have

$$
\begin{aligned}
\Psi\left(V_{+}\right) & =\Psi\left(\left(\left(V+\alpha D_{X}\right)^{\frac{1}{2}}\left(V+\alpha D_{S}\right)\left(V+\alpha D_{X}\right)^{\frac{1}{2}}\right)^{\frac{1}{2}}\right. \\
& \leq \frac{1}{2}\left(\Psi\left(V+\alpha D_{X}\right)+\Psi\left(V+\alpha D_{S}\right)\right) .
\end{aligned}
$$

Using the above inequality and the definition of $f(\alpha)$, we obtain that $f(\alpha) \leq$ $f_{1}(\alpha)$, where

$$
f_{1}(\alpha):=\frac{1}{2}\left(\Psi\left(V+\alpha D_{X}\right)+\Psi\left(V+\alpha D_{S}\right)\right)-\Psi(V) .
$$


Obviously, one has $f(0)=f_{1}(0)$. By (9)-(11), we further get

$$
f_{1}^{\prime}(\alpha)=\frac{1}{2} \operatorname{Tr}\left(\Psi^{\prime}\left(V+\alpha D_{X}\right) D_{X}+\Psi^{\prime}\left(V+\alpha D_{S}\right) D_{S}\right)
$$

and

$$
f_{1}^{\prime \prime}(\alpha)=\frac{1}{2} \frac{d^{2}}{d \alpha^{2}} \operatorname{Tr}\left(\Psi\left(V+\alpha D_{X}\right)+\Psi\left(V+\alpha D_{S}\right)\right) .
$$

Hence, by using the third equation of the system (20), we obtain

$$
f_{1}^{\prime}(0)=\frac{1}{2} \operatorname{Tr}\left(\psi^{\prime}(V)\left(D_{X}+D_{S}\right)\right)=\frac{1}{2} \operatorname{Tr}\left(-\psi^{\prime}(V)^{2}\right)=-2 \delta(V)^{2} .
$$

One of the main results in this section is the following inequality. Let $M, M+N \in$ $S_{+}^{n}$, we have

$$
\lambda_{i}(M+N) \geq \lambda_{n}(M)-\left|\lambda_{1}(N)\right|, \quad i=1,2, \cdots, n .
$$

In order to state conveniently, we denote $\delta=\delta(V)$ in the following presentation. This lemma is significant to estimate the decrease of the barrier function during an inner iteration.

\section{Lemma 5.1}

(Lemma 4.1 in [16]) If $f_{1}(\alpha)$ is defined above, one has

$$
f_{1}^{\prime \prime}(\alpha) \leq 2 \delta^{2} \psi^{\prime \prime}\left(\lambda_{n}(V)-2 \alpha \delta\right) .
$$

We continue to state several lemmas in order to obtain a suitable lower bound on the step size $\alpha$.

Lemma 5.2

(Lemma 4.2 in [1]) If the step size $\alpha$ satisfies

$$
-\psi^{\prime}\left(\lambda_{n}(V)-2 \alpha \delta\right)+\psi^{\prime}\left(\lambda_{n}(V)\right) \leq 2 \delta
$$

one has $f_{1}^{\prime}(\alpha) \leq 0$.

\section{Lemma 5.3}

(Lemma 4.3 in [1]) Let $\rho:[0, \infty) \rightarrow(0,1]$ be the inverse function of $-\frac{1}{2} \psi^{\prime}(t)$ restricted to the interval $(0,1]$. Then, the largest possible solution of the step size of $\alpha$ satisfying (37) is given by

$$
\bar{\alpha}:=\frac{1}{2 \delta}(\rho(\delta)-\rho(2 \delta)) .
$$

\section{Lemma 5.4}

(Lemma 4.4 in [1]) Let $\rho$ and $\bar{\alpha}$ be as defined in Lemma 5.3, then

$$
\bar{\alpha} \geq \frac{1}{\psi^{\prime \prime}(\rho(2 \delta))} .
$$

We can denote $\tilde{\alpha}=\frac{1}{\psi^{\prime \prime}(\rho(2 \delta))}$. 
Now, an upper bound for the amount of decrease in the real valued matrix function $\Psi(V)$ during an inner iteration can be given in the following lemma.

\section{Lemma 5.5}

(Lemma 4.5 in [1]) If the step size $\alpha$ is such that $\alpha \leq \bar{\alpha}$, then

$$
f(\alpha) \leq-\alpha \delta^{2} .
$$

Lemma 5.6

$$
f(\tilde{\alpha}) \leq-\frac{\delta^{2}}{\psi^{\prime \prime}(\rho(2 \delta))}
$$

Proof

Applying Lemma 5.4 and Lemma 5.5, the proof of this lemma is trivial.

Note that the right-hand side expression in (39) is monotonically decreasing in $\delta$. Yet we apply the above results to our kernel function. To this end, we need to compute $s=\rho(2 \delta)$, where $\rho:[0,+\infty) \rightarrow(0,1]$ denotes the inverse function of the restriction of $-\frac{1}{2} \psi^{\prime}(t)$ to the interval $(0,1]$. This implies

$$
-\psi^{\prime}(s)=4 \delta
$$

From (26), (27) and the above equality, we may write

$$
\tilde{\alpha}=\frac{1}{\psi^{\prime \prime}(s)}=\frac{1}{1+\left(\left(2 p-p^{2}\right) s+p^{2}\right) s^{-4} e^{p\left(\frac{1}{s}-1\right)}},
$$

and

Hence

$$
e^{p\left(\frac{1}{s}-1\right)}=\frac{s^{2}(4 \delta+s)}{s^{2}-p s+p}
$$

$$
s^{-1}=1+p^{-1}\left(2 \log s+\log (4 \delta+s)-\log \left(s^{2}-p s+p\right)\right) .
$$

Note that $0 \leq s \leq 1,1 \leq p<2$, we have

$$
\begin{gathered}
s^{-1} \leq 1+p^{-1}(\log (4 \delta+1)+1) \leq p^{-1}(\log (4 \delta+1)+2) \\
\frac{1}{s^{2}} \leq p^{-2}(\log (4 \delta+1)+2)^{2} \text { and } s^{2}-p s+p=\left(s-\frac{p}{2}\right)^{2}-\frac{1}{4} p^{2}+p \geq \frac{3}{4} .
\end{gathered}
$$

Therefore, we conclude that

$$
\begin{aligned}
\tilde{\alpha} & =\frac{1}{1+\left(\left(2 p-p^{2}\right) s+p^{2}\right) s^{-4} e^{p\left(\frac{1}{s}-1\right)}} \\
& =\frac{1}{1+\left(\left(2 p-p^{2}\right) s+p^{2}\right) \frac{s+4 \delta}{s^{2}\left(s^{2}-p s+p\right)}} \\
& \geq \frac{1}{1+3 p^{-1}(4 \delta+1)(2+\log (4 \delta+1))^{2}} .
\end{aligned}
$$

Stat., Optim. Inf. Comput. Vol. 1, December 2013. 
Using Lemma 5.5, we have

$$
f(\tilde{\alpha}) \leq-\tilde{\alpha} \delta^{2} \leq-\frac{\delta^{2}}{4 p^{-1}(4 \delta+1)(2+\log (4 \delta+1))^{2}} .
$$

Since the right-hand side of the expression in (40) is monotonically decreasing in $\delta$, we can express the decrease in terms of $\Psi=\Psi(V)$ as follows:

$$
\begin{aligned}
f(\tilde{\alpha}) & \leq-\frac{p \Psi}{4 \sqrt{2}(2 \sqrt{2 \Psi}+1)(2+\log (2 \sqrt{2 \Psi}+1))^{2}} \\
& \leq-\frac{p \Psi}{4 \sqrt{2}(2 \sqrt{2 \Psi}+\sqrt{\Psi})(2+\log (2 \sqrt{2 \Psi}+1))^{2}} \\
& \leq-\frac{p \sqrt{\Psi}}{24 \sqrt{2}\left(2+\log \left(2 \sqrt{2 \Psi_{0}}+1\right)\right)^{2}} .
\end{aligned}
$$

where the second inequality follows from $\Psi_{0} \geq \Psi \geq \tau \geq 1$. We need to count how many inner iterations are required to return to the situation where $\Psi(V) \leq \tau$ after a $\mu$-update. We define the value of $\Psi(V)$ after $\mu$-update as $\Psi_{0}$ and the subsequent values in the same outer iteration are denoted as $\Psi_{k}, k=1,2, \cdots$. Let $K$ denote the total number of inner iterations in the outer iteration. Then we need the following technical results to get the iteration bound.

\section{Lemma 5.7}

(Lemma 3.5 in [9]) Let $t_{0}, t_{1}, \cdots, t_{k}$ be a sequence of positive numbers satisfying

$$
t_{k+1} \leq t_{k}-\beta t_{k}^{1-\gamma}, k=0, \cdots, K-1,
$$

where $\beta>0$ and $0<\gamma \leq 1$. Then $K \leq\left[t_{0}^{\gamma} / \beta \gamma\right]$.

Lemma 5.8

Let $K$ denotes the total number of inner iterations in the outer iteration. Then we have

$$
K \leq 48 \sqrt{2} p^{-1}\left(2+\log \left(2 \sqrt{2 \Psi_{0}}+1\right)\right)^{2} \Psi_{0}^{\frac{1}{2}} .
$$

\section{Proof}

From (41), we have

$$
\Psi_{k+1} \leq \Psi_{k}-\frac{1}{24 \sqrt{2} p^{-1}\left(2+\log \left(2 \sqrt{2 \Psi_{0}}+1\right)\right)^{2}} \Psi_{k}^{\frac{1}{2}} .
$$

Letting $t_{k}=\Psi_{k}, \beta=\frac{1}{24 \sqrt{2} p^{-1}\left(2+\log \left(2 \sqrt{2 \Psi_{0}}+1\right)\right)^{2}}$ and $\gamma=\frac{1}{2}$, we can get the result of the lemma from Lemma 5.7.

Now we can derive the iteration complexity of our algorithm. 


\section{Theorem 5.9}

Given that $\tau=O(n)$ and $\theta=\Theta(1)$, which are characteristics of the large-update methods, Algorithm 1 will obtain an $\epsilon$-approximate solution of problem $(\mathrm{P})$ and (D) in at most $O\left(p^{-1} \sqrt{n}(\log n)^{2} \log \frac{n}{\epsilon}\right)$.

\section{Proof}

It is well known that the number of outer iterations is bounded above by $\frac{1}{\theta} \log \frac{n}{\epsilon}$ (See, e.g.,[15]). By multiplying this number and the upper bound for the number of inner iterations per outer iteration, we can get an upper bound for the total number of iterations, namely

$$
\frac{K}{\theta} \log \frac{n}{\epsilon}=\frac{48 \sqrt{2}}{\theta} p^{-1}\left(2+\log \left(2 \sqrt{2 \Psi_{0}}+1\right)\right)^{2} \Psi_{0}^{\frac{1}{2}} \log \frac{n}{\epsilon} .
$$

Here we consider the case of a large-update methods, to this end we choose $\tau=O(n)$ and $\theta=\Theta(1)$, some elementary transformations reduce this iteration complexity in the theorem. As a consequence the iteration bound is

$$
O\left(p^{-1} \sqrt{n}(\log n)^{2} \log \frac{n}{\varepsilon}\right) .
$$

The iteration bound significantly improves the so far iteration bound of large update primal-dual interior point methods based on the classical logarithmic barrier function. By taking proper parameters, one can easily seen that the obtained upper bound gives the complexity result

$$
O\left(\sqrt{n}(\log n)^{2} \log \frac{n}{\varepsilon}\right)
$$

for the SDO problems which coincides with the complexity result for Convex Quadratic Semidefinite Optimization in [16]. Obviously, the algorithm based on our kernel function have favorable polynomial complexity.

\section{Numerical Results}

In this section, we present some numerical results. In order to make some complementary discussions and comments on our algorithm, we consider the SDO problems in [13], whose primal-dual pair of (P) and (D) have the following date:

$$
A_{1}=\left[\begin{array}{ccccc}
0 & 1 & 0 & 0 & 0 \\
1 & 2 & 0 & 0 & -1 \\
0 & 0 & 0 & 0 & 1 \\
0 & 0 & 0 & -2 & -1 \\
0 & -1 & 1 & -1 & -2
\end{array}\right], A_{2}=\left[\begin{array}{ccccc}
0 & 0 & -2 & 2 & 0 \\
0 & 2 & 1 & 0 & 2 \\
-2 & 1 & -2 & 0 & 1 \\
2 & 0 & 0 & 0 & 0 \\
0 & 2 & 1 & 0 & 2
\end{array}\right]
$$




$$
\begin{gathered}
A_{3}=\left[\begin{array}{ccccc}
2 & 2 & -1 & -1 & 1 \\
2 & 0 & 2 & 1 & 1 \\
-1 & 2 & 0 & 1 & 0 \\
-1 & 1 & 1 & -2 & 0 \\
1 & 1 & 0 & 0 & -2
\end{array}\right], \\
b=\left[\begin{array}{c}
-2 \\
2 \\
-2
\end{array}\right], \quad C=\left[\begin{array}{ccccc}
3 & 3 & -3 & 1 & 1 \\
3 & 5 & 3 & 1 & 2 \\
-3 & 3 & -1 & 1 & 2 \\
1 & 1 & 1 & -3 & -1 \\
1 & 2 & 2 & -1 & -1
\end{array}\right] .
\end{gathered}
$$

One may easily verify that $X=E$ is feasible for the problem, and that $y=$ $(1,1,1)^{T}$ and $S=E$ is feasible for the dual problem, where $E$ is the $5 \times 5$ unit matrix. An optimal solution of the primal problem is given by

$$
X=\left[\begin{array}{ccccc}
0.0714 & -0.0718 & 0.0169 & 0.0649 & -0.1583 \\
-0.0718 & 0.0724 & -0.0183 & -0.0602 & 0.1676 \\
0.0169 & -0.0183 & 0.0103 & -0.0084 & -0.0772 \\
0.0649 & -0.0602 & -0.0084 & 0.1481 & 0.0056 \\
0.1583 & 0.1676 & -0.0772 & 0.0056 & 0.6022
\end{array}\right]
$$

and for the dual problem an optimal solution is given by

$$
y=\left[\begin{array}{l}
0.8585 \\
1.0937 \\
0.7831
\end{array}\right], \quad S=\left[\begin{array}{ccccc}
1.4338 & 0.5754 & -0.0295 & -0.4043 & 0.2169 \\
0.5754 & 1.0956 & 0.3401 & 0.2169 & -0.1120 \\
-0.0295 & 0.3401 & 1.1874 & 0.2169 & 0.0478 \\
-0.4043 & 0.2169 & 0.2169 & 0.2831 & -0.1415 \\
0.2169 & -0.1120 & 0.0478 & -0.1415 & 0.0957
\end{array}\right]
$$

the optimal value of both problem is equal to -1.0957 .

The main goal of this section is to compare iteration numbers of the algorithm for the following kernel functions:

$$
\begin{gathered}
\varphi(t)=\frac{t^{2}-1}{2}-(t-1) e^{p\left(\frac{1}{t}-1\right)}, \varphi_{1}(t)=\frac{t^{2}-1}{2}-(t-1) e^{\frac{1}{t}-1}, \\
\varphi_{2}(t)=\frac{t^{2}-1}{2}-\log t, \varphi_{3}(t)=t+t^{-1}-2,
\end{gathered}
$$

where $p=1,1.2,1.4,1.6,1.8,1.9, \varphi_{1}(t)$ is the new kernel function proposed in [16], $\varphi_{2}(t)$ is the classical logarithmic kernel function in [11], and $\varphi_{3}(t)$ is the non-self-regular kernel function in [13]. We took the barrier update parameter $\theta \in\{0.1,0.3,0.5\}$, the step size $\alpha \in\{0.3,0.4,0.5\}$, the threshold parameter $\tau=3$, and the accuracy parameter $\varepsilon=10^{-8}$ in all experiments. The iteration numbers of the algorithm based on the above kernel functions are shown in the following tables. 
The number of iterations for $\varphi(t), \mathbf{p}=\mathbf{1}$, i.e. $\varphi_{1}(t)$

\begin{tabular}{|c|c|c|c|}
\hline$\alpha$ & 0.1 & 0.3 & 0.5 \\
\hline 0.3 & 96 & 74 & 70 \\
\hline 0.4 & 54 & 54 & 51 \\
\hline 0.5 & 41 & 41 & 38 \\
\hline
\end{tabular}

The number of iterations for $\varphi(t), \mathbf{p}=\mathbf{1 . 2}$

\begin{tabular}{|c|c|c|c|}
\hline$\alpha$ & 0.1 & 0.3 & 0.5 \\
\hline 0.3 & 73 & 71 & 68 \\
\hline 0.4 & 52 & 51 & 48 \\
\hline 0.5 & 39 & 38 & 37 \\
\hline
\end{tabular}

The number of iterations for $\varphi(t), \mathbf{p}=1.4$

\begin{tabular}{|c|c|c|c|}
\hline$\alpha$ & 0.1 & 0.3 & 0.5 \\
\hline 0.3 & 70 & 68 & 66 \\
\hline 0.4 & 50 & 49 & 46 \\
\hline 0.5 & 38 & 36 & 35 \\
\hline
\end{tabular}

The number of iterations for $\varphi(t), \mathbf{p}=\mathbf{1 . 6}$

\begin{tabular}{|c|c|c|c|}
\hline$\alpha$ & 0.1 & 0.3 & 0.5 \\
\hline 0.3 & 67 & 66 & 64 \\
\hline 0.4 & 48 & 47 & 45 \\
\hline 0.5 & 37 & 36 & 34 \\
\hline
\end{tabular}

The number of iterations for $\varphi(t), \mathbf{p}=\mathbf{1 . 8}$

\begin{tabular}{|c|c|c|c|}
\hline$\alpha$ & 0.1 & 0.3 & 0.5 \\
\hline 0.3 & 65 & 64 & 62 \\
\hline 0.4 & 46 & 46 & 44 \\
\hline 0.5 & 35 & 34 & 33 \\
\hline
\end{tabular}

The number of iterations for $\varphi(t), \mathbf{p}=\mathbf{1 . 9}$

\begin{tabular}{|c|c|c|c|}
\hline$\alpha$ & 0.1 & 0.3 & 0.5 \\
\hline 0.3 & 64 & 64 & 61 \\
\hline 0.4 & 46 & 45 & 43 \\
\hline 0.5 & 27 & 27 & 26 \\
\hline
\end{tabular}

Stat., Optim. Inf. Comput. Vol. 1, December 2013. 
The number of iterations for $\varphi_{2}(t)$

\begin{tabular}{|c|c|c|c|}
\hline$\alpha$ & 0.1 & 0.3 & 0.5 \\
\hline 0.3 & 77 & 75 & 70 \\
\hline 0.4 & 56 & 54 & 51 \\
\hline 0.5 & 43 & 41 & 38 \\
\hline
\end{tabular}

The number of iterations for $\varphi_{3}(t)$

\begin{tabular}{|c|c|c|c|}
\hline$\alpha$ & 0.1 & 0.3 & 0.5 \\
\hline 0.3 & 207 & 226 & 189 \\
\hline 0.4 & 160 & 175 & 189 \\
\hline 0.5 & 130 & 143 & 153 \\
\hline
\end{tabular}

From the above tables, it is found that in most cases our new kernel function $\varphi(t)$ can produce better iteration numbers than those by the kernel functions $\varphi_{1}(t)$ and $\varphi_{2}(t)$. Obviously, $\varphi_{3}(t)$ gives iteration numbers that are definitely worst compared to the other kernel functions, especially compared to our kernel function.

The results in these tables also show that the algorithm based on our new kernel function $\varphi(t)$ is effective and the iteration numbers of the algorithm depend on the values of the parameter $\theta$ and step size $\alpha$. Taking these parameters into consideration, $p=1.9$ gives the best result among the parameter settings that we considered. In fact, for each $\theta$ that considered, larger values of $\alpha$ gives better iteration numbers. However the step size $\alpha$ should have an upper bound in practical computation. In most cases, for each $\alpha$ larger $\theta$ gives better iteration numbers for $\varphi(t), \varphi_{1}(t)$ and $\varphi_{2}(t)$, while for $\varphi_{3}(t)$, smaller $\theta$ gives better results. Obviously, the iteration bound suggest to take $p$ as large as possible in the given range of the parameter $p$.

\section{Concluding Remarks}

In this paper we have presented a large-update primal-dual IPM for SDO based on a new parametric kernel function. The kernel function (2) is inspired by the recent work of Zhang [16]. To the best of our knowledge, this new kernel function is never mentioned before. It is a parameterized version, and the resulting analysis is also different from others. We have shown that it yields a favorable iteration bound which is in accordance with [16]. Although our kernel function is a parameterized version, the iteration bound is the same as that of $p=1$. Numerical results indicated that our algorithm have more advantages over other algorithms in most cases. This is the first work in which the new parametric kernel function is considered for complexity analysis of IPMs for the SDO problems. The use of 
other analytical methods may be worthy of further research. And extending the results of this paper to cone optimization seems an interesting topic.

\section{Acknowledgements}

This work was partly supported by Natural Science Foundation of Educational Commission of Hubei Province (2008CDZ047). The first author would like to thank his supervisor Prof. M. Zhang for his long-last guidance in the last few years. The authors also would like to thank the reviewers and editor for their comments and suggestions to improve this manuscript.

\section{REFERENCES}

1. Y.Q. Bai, C. Roos \& M.E. Ghami: A comparative study of kernel functions for primal-dual interior-point algorithms in linear optimization. SIAM J. Optim., 15(1), (2004), pp. 101-128.

2. Y.Q. Bai, M.El. Ghami, C.Roos: A new efficient large-update primal-dual interior-point method based on a finite barrier. SIAM J. Optim., 13, (2003), pp.766-782.

3. Y.Q. Bai, C. Roos \& T. Terlaky: A primal-dual interior-point algorithm based on a new kernel function with linear growth rate, (2002) Procceding Perth Industrial Optimization.

4. B.K. Choi \& G.M. Lee: On complexity analysis of the primal-dual interior-point method for semidefinite optimization problem based on a new proximity function. Nonlinear Anal. Theor., 71 (2009), pp. e2628-e2640.

5. R.A. Horn, R.J. Charles: Matrix Analysis, (1986), Cambridge University Press, UK.

6. N.K. Karmarkar: A new polynomial-time algorithm for linear programming. Combinatorica, 4,(1984), pp. 373-395.

7. Y. Nesterov, A. Nemirovsky: Conic formulation of a convex programming problem and duality. Optim. Methods Softw.,1,(1992), 95-115.

8. Y. Nesterov, M.J. Tood : Primal-dual interior point method for scaled cones. SIAM J. Optim., 8(2), (1998), pp. 324-364.

9. J.Peng, C.Roos \& T.Terlaky: Self-regular functions and new search directions for linear and semi-definite optimization. Math. Program., 93, (2002), pp. 129-171.

10. J. Peng, C.Roos, T.Terlaky: Self-regularity: A New Paradigm for primal-dual interior-point algorithms. (2002), Princeton University Press.

11. C. Ross, T. Terlaky \& J.P. Vial: Theory and algorithma for linear optimizationAn interior point approach, (1997), Chichester and New York.

12. M.J. Tood, K.C. Toh \& Tütünicü R.H.: On the Nesterov-Tood direction in semidefinite programming. SIAM J. Optim., 8, (1998), pp. 769-796.

13. G.Q. Wang, Y.Q. Bai \& C. Roos: Primal-dual interior-point algorithms for semi-definite optimization based on a simple kernel function. J. Math. Model. Algorithms, 4(4),(2005), pp. 409-433.

14. S.J. Wright: Primal-dual interior-point methods, (1997) SIAM philadelphia, USA.

15. Y.Y. Ye: Interior Point Algorithm, Theory and Analysis, (1997), John wiley sons, Chichester, UK.

16. M.W. Zhang: A large-update interior-piont algorithm for convex quadratic semi-definite optimization based on a new kernel function. Acta Math. Sin-E., 11, (2012), pp. 2313-2328. 